\title{
Positive solutions for a boundary value problem of fractional differential equation with $p$-Laplacian operator
}

\author{
Yuansheng Tian' ${ }^{1}$ Zhanbing Bai ${ }^{* *}$ (D) and Sujing Sun²
}

${ }^{*}$ Correspondence:
zhanbingbai@163.com
${ }^{2}$ College of Mathematics and
System Sciences, Shandong
University of Science and
Technology, Qingdao, P.R. China
Full list of author information is
available at the end of the article

\section{correspondence:}

available at the end of the article

\begin{abstract}
In this paper, the existence of a positive solution to a boundary value problem of fractional differential equations with the $p$-Laplacian operator is studied. By applying a monotone iterative method, some existence results of positive solutions are obtained. In addition, an example is included to illustrate the main results.
\end{abstract}

MSC: 34A08; 34B18; 35J05

Keywords: Fractional differential equation; Boundary value problem; $p$-Laplacian operator; Positive solution

\section{Introduction}

In this paper, we consider the following boundary value problem of fractional differential equations with a $p$-Laplacian operator:

$$
\begin{aligned}
& D^{\gamma}\left(\phi_{p}\left(D^{\alpha} u(t)\right)\right)=f(t, u(t)), \quad 0<t<1, \\
& u(0)=D^{\alpha} u(0)=0, \quad D^{\beta} u(1)=a D^{\beta} u(\xi), \quad D^{\alpha} u(1)=b D^{\alpha} u(\eta),
\end{aligned}
$$

where $\alpha, \beta, \gamma \in \mathbb{R} ; 1<\alpha, \gamma \leq 2 ; \beta>0$ and $1+\beta \leq \alpha ; \xi, \eta \in(0,1) ; a, b \in[0,+\infty) ; 1-$ $a \xi^{\alpha-\beta-1}>0 ; 1-b^{p-1} \eta^{\gamma-1}>0$ and $\phi_{p}(s)=|s|^{p-2} s, p>1, \phi_{q}=\left(\phi_{p}\right)^{-1}, \frac{1}{p}+\frac{1}{q}=1, D^{\alpha}$ is the Riemann-Liouville differentiation and $f \in C([0,1] \times[0,+\infty),[0,+\infty))$.

Fractional differential equations arise in many engineering and scientific disciplines as the mathematical modeling of systems and processes in the fields of physics, chemistry, aerodynamics, electrodynamics of a complex medium, polymer rheology, etc. Fractional differential equations also serve as an excellent tool for the description of hereditary properties of various materials and processes. In consequence, fractional differential equations have been of great interest. For details, see fractional two-point boundary value problems $[29,31,32,35,57,64]$, fractional boundary value problems at resonance $[5,8,67,69,71]$, fractional multi-point problems with nonresonance [5, 8, 44, 48, 58, 61, 68], fractional initial value problems $[6,7,34]$, fractional impulsive problems $[48,72]$, fractional integral boundary value problems $[14,40,46,62]$, fractional $p$-Laplace problems $[15,18,20-22,28$, $36,39,45,47,49,50,52,60,65,66,70]$, fractional problems with lower and upper solution 
[7, 39, 51, 59], fractional control problems, [41, 43, 53-56], fractional soliton problems $[19,24,26,42]$, fractional singular problems [17, 27, 30, 37, 38, 63].

On the other hand, the differential equations with $p$-Laplacian operator arise in the modeling of different physical and natural phenomena, non-Newtonian mechanics, nonlinear elasticity and glaciology, population biology, nonlinear flow laws, and system of Monge-Kantorovich partial differential equations. There are a very large number of papers devoted to the existence of solutions of the $p$-Laplacian operator $[15,18,20-22,28$, $36,39,45,47,49,50,52,60,65,66]$. The approaches are mainly topological, fixed-point and continuation theorems, degree and fixed-point index theory.

In this paper, we study the existence of positive solutions for boundary value problem (1.1), by applying a monotone iterative method, some existence results of positive solutions are obtained. In the light of the above and to the best $o$ four knowledge, this is the first study which discuss fractional $p$-Laplacian with lower and upper solution method. Taking into account that sometimes the corresponding research about the Riesz fractional derivative is interesting, see $[16,25]$, in the future work, we will focus our concentration on the Riesz derivative. Also, the reader can find some new methods for approximate solutions of fractional integro-differential equations involving the Caputo-Fabrizio derivative or extended fractional Caputo-Fabrizio derivative $[1-4,9-13,23,33]$. The approximation solutions are interesting and need more concentration.

The organization of this paper is as follows. In Sect. 2, we present some necessary definitions from fractional calculus theory. In Sect. 3, we prove the main results about the existence of positive solution of the boundary value problem (1.1). In Sect. 4, we will give an example to illustrate our main results.

\section{Preliminaries}

In this section, we present some necessary definitions from fractional calculus theory.

Definition 2.1 ([32]) The Riemann-Liouville fractional integral of a function $x:(0$, $+\infty) \rightarrow \mathbb{R}$ of order $\alpha>0$ is given by

$$
I^{\alpha} x(t)=\frac{1}{\Gamma(\alpha)} \int_{0}^{t}(t-s)^{\alpha-1} x(s) d s
$$

provided the right side is pointwise defined on $(0, \infty)$.

Definition 2.2 ([32]) The Riemann-Liouville fractional derivative of order $\alpha>0$ of a continuous function $x:(0,+\infty) \rightarrow \mathbb{R}$ is given by

$$
D^{\alpha} x(t)=\frac{1}{\Gamma(n-\alpha)}\left(\frac{d}{d t}\right)^{n} \int_{0}^{t} \frac{x(s)}{(t-s)^{\alpha-n+1}} d s
$$

where $n=[\alpha]+1,[\alpha]$ denotes the integer part of number $\alpha$, provided the right side is pointwise defined on $(0, \infty)$.

Lemma 2.1 ([32])

(1) If $x \in L(0,1), \rho>\sigma>0$, then

$$
D^{\sigma} I^{\rho} x(t)=I^{\rho-\sigma} x(t), \quad D^{\sigma} I^{\sigma} x(t)=x(t) .
$$


(2) If $\rho>0, \lambda>0$, then

$$
D^{\rho} t^{\lambda-1}=\frac{\Gamma(\lambda)}{\Gamma(\lambda-\rho)} t^{\lambda-\rho-1}
$$

Lemma 2.2 ([32]) Assume that $x \in C(0,1) \cap L(0,1)$ with a fractional derivative of order $\alpha>0$ that belongs to $C(0,1) \cap L(0,1)$. Then

$$
I^{\alpha} D^{\alpha} x(t)=x(t)+c_{1} t^{\alpha-1}+c_{2} t^{\alpha-2}+\cdots+c_{N} t^{\alpha-N}, \quad c_{i} \in \mathbb{R}, i=1,2, \ldots, N,
$$

where $N$ is the smallest integer greater than or equal to $\alpha$.

\section{Main results}

In this section, we consider the existence of positive solution for problem (1.1).

Lemma 3.1 If $h \in C[0,1], \alpha \in(1,2], \beta>0$ and $1+\beta \leq \alpha, \xi \in(0,1), a \in[0,+\infty), \mathscr{A}:=$ $a \xi^{\alpha-\beta-1}$, then the boundary value problem

$$
\begin{aligned}
& D^{\alpha} u(t)+h(t)=0, \quad 0<t<1, \\
& u(0)=0, \quad D^{\beta} u(1)=a D^{\beta} u(\xi),
\end{aligned}
$$

has an unique solution

$$
u(t)=\int_{0}^{1} G(t, s) h(s) d s
$$

where

$$
G(t, s)=\left\{\begin{array}{l}
\frac{\alpha^{\alpha-1}(1-s)^{\alpha-\beta-1}-(1-\mathscr{A})(t-s)^{\alpha-1}-a t^{\alpha-1}(\xi-s)^{\alpha-\beta-1}}{\Gamma(\alpha)(1-\mathscr{A})}, \\
0 \leq s \leq t \leq 1, s \leq \xi, \\
\frac{t^{\alpha-1}(1-s)^{\alpha-\beta-1}-(1-\mathscr{A})(t-s)^{\alpha-1}}{\Gamma(\alpha)(1-\mathscr{A})}, \quad 0<\xi \leq s \leq t \leq 1, \\
\frac{t^{\alpha-1}(1-s)^{\alpha-\beta-1}-a t^{\alpha-1}(\xi-s)^{\alpha-\beta-1}}{\Gamma(\alpha)(1-\mathscr{A})}, \quad 0 \leq t \leq s \leq \xi<1, \\
\frac{t^{\alpha-1}(1-s)^{\alpha-\beta-1}}{\Gamma(\alpha)(1-\mathscr{A})}, \quad 0 \leq t \leq s \leq 1, \xi \leq s .
\end{array}\right.
$$

Proof By applying Lemma 2.2, we can reduce Eq. (3.1) to an equivalent integral equation

$$
u(t)=-I^{\alpha} h(t)+c_{1} t^{\alpha-1}+c_{2} t^{\alpha-2},
$$

for some $c_{1}, c_{2} \in \mathbb{R}$. Note that $u(0)=0$, we have $c_{2}=0$. Consequently the general solution of Eq. (3.1) is

$$
u(t)=-I^{\alpha} h(t)+c_{1} t^{\alpha-1} .
$$

By (3.6) and Lemma 2.1, we have

$$
D^{\beta} u(t)=-D^{\beta} I^{\alpha} h(t)+c_{1} D^{\beta} t^{\alpha-1}=-I^{\alpha-\beta} h(t)+c_{1} \frac{\Gamma(\alpha)}{\Gamma(\alpha-\beta)} t^{\alpha-\beta-1} .
$$


So,

$$
\begin{aligned}
& D^{\beta} u(1)=-\int_{0}^{1} \frac{(1-s)^{\alpha-\beta-1}}{\Gamma(\alpha-\beta)} h(s) d s+c_{1} \frac{\Gamma(\alpha)}{\Gamma(\alpha-\beta)} \\
& D^{\beta} u(\xi)=-\int_{0}^{\xi} \frac{(\xi-s)^{\alpha-\beta-1}}{\Gamma(\alpha-\beta)} h(s) d s+c_{1} \frac{\Gamma(\alpha)}{\Gamma(\alpha-\beta)} \xi^{\alpha-\beta-1} .
\end{aligned}
$$

By $D^{\beta} u(1)=a D^{\beta} u(\xi)$, combining with (3.7) and (3.8), we obtain

$$
c_{1}=\frac{1}{\Gamma(\alpha)(1-\mathscr{A})}\left\{\int_{0}^{1}(1-s)^{\alpha-\beta-1} h(s) d s-a \int_{0}^{\xi}(\xi-s)^{\alpha-\beta-1} h(s) d s\right\} .
$$

So, the unique solution of the problem (3.1), (3.2) is

$$
\begin{aligned}
u(t)= & -\int_{0}^{t} \frac{(t-s)^{\alpha-1}}{\Gamma(\alpha)} h(s) d s+\frac{t^{\alpha-1}}{\Gamma(\alpha)(1-\mathscr{A})}\left\{\int_{0}^{1}(1-s)^{\alpha-\beta-1} h(s) d s\right. \\
& \left.-a \int_{0}^{\xi}(\xi-s)^{\alpha-\beta-1} h(s) d s\right\} \\
= & \int_{0}^{1} G(t, s) h(s) d s .
\end{aligned}
$$

The proof is completed.

Lemma 3.2 If $h \in C[0,1], \phi_{p}(s)=|s|^{p-2} s, p>1, \phi_{q}=\left(\phi_{p}\right)^{-1}, \frac{1}{p}+\frac{1}{q}=1, \alpha, \beta, \gamma \in \mathbb{R}, 1<\alpha$, $\gamma \leq 2, \beta>0$ and $1+\beta \leq \alpha, 0<\xi, \eta<1, a, b \in[0,+\infty), \mathscr{B}=: b^{p-1} \eta^{\gamma-1}$, then the problem

$$
\begin{aligned}
& D^{\gamma}\left(\phi_{p}\left(D^{\alpha} u(t)\right)\right)=h(t), \quad 0<t<1, \\
& u(0)=D^{\alpha} u(0)=0, \quad D^{\beta} u(1)=a D^{\alpha} u(\xi), \quad D^{\alpha} u(1)=b D^{\alpha} u(\eta),
\end{aligned}
$$

has an unique solution

$$
u(t)=\int_{0}^{1} G(t, s) \phi_{q}\left(\int_{0}^{1} H(s, \tau) h(\tau) d \tau\right) d s
$$

where

$$
H(t, s)= \begin{cases}\frac{[t(1-s)]^{\gamma-1}-b^{p-1}[t(\eta-s)]^{\gamma-1}-(1-\mathscr{B})(t-s)^{\gamma-1}}{(1-\mathscr{B}) \Gamma(\gamma)}, & 0 \leq s \leq t \leq 1, s \leq \eta \\ \frac{[t(1-s)]^{\gamma-1}-(1-\mathscr{B})(t-s) \gamma^{\gamma-1}}{(1-\mathscr{O}) \Gamma(\gamma)}, & 0<\eta \leq s \leq t \leq 1 ; \\ \frac{[t(1-s)]^{\gamma-1}-b^{p-1}[t(\eta-s)]^{\gamma-1}}{(1-\mathscr{B}) \Gamma(\gamma)}, & 0 \leq t \leq s \leq \eta<1 ; \\ \frac{[t(1-s))^{\gamma-1}}{(1-\mathscr{B}) \Gamma(\gamma)}, & 0 \leq t \leq s \leq 1, \eta \leq s .\end{cases}
$$

$G(t, s)$ is defined by (3.4).

Proof From Eq. (3.9), and Lemma 2.2, we have

$$
\phi_{p}\left(D^{\alpha} u(t)\right)=I^{\gamma} h(t)+d_{1} t^{\gamma-1}+d_{2} t^{\gamma-2},
$$


for some $d_{1}, d_{2} \in \mathbb{R}$. Note that $D^{\beta} u(0)=0$, we have $d_{2}=0$, then

$$
\phi_{p}\left(D^{\alpha} u(t)\right)=\frac{1}{\Gamma(\gamma)} \int_{0}^{t}(t-\tau)^{\gamma-1} h(\tau) d \tau+d_{1} t^{\gamma-1}
$$

So,

$$
\begin{aligned}
& \phi_{p}\left(D^{\alpha} u(1)\right)=\frac{1}{\Gamma(\gamma)} \int_{0}^{1}(1-\tau)^{\gamma-1} h(\tau) d \tau+d_{1}, \\
& \phi_{p}\left(D^{\alpha} u(\eta)\right)=\frac{1}{\Gamma(\gamma)} \int_{0}^{\eta}(\eta-\tau)^{\gamma-1} h(\tau) d \tau+d_{1} \eta^{\gamma-1} .
\end{aligned}
$$

By $D^{\alpha} u(1)=b D^{\alpha} u(\eta)$, combining with (3.14) and (3.15), we have

$$
d_{1}=-\int_{0}^{1} \frac{(1-\tau)^{\gamma-1}}{\Gamma(\gamma)(1-\mathscr{B})} h(\tau) d \tau+\int_{0}^{\eta} \frac{b^{p-1}(\eta-\tau)^{\gamma-1}}{\Gamma(\gamma)(1-\mathscr{B})} h(\tau) d \tau .
$$

So, the unique solution of problem (3.1), (3.2) is

$$
\begin{aligned}
\phi_{p}\left(D^{\alpha} u(t)\right)= & \int_{0}^{t} \frac{(t-\tau)^{\gamma-1}}{\Gamma(\gamma)} h(\tau) d \tau-\int_{0}^{1} \frac{t^{\gamma-1}(1-\tau)^{\gamma-1}}{\Gamma(\gamma)(1-\mathscr{B})} h(\tau) d \tau \\
& +\int_{0}^{\eta} \frac{b^{p-1} t^{\gamma-1}(\eta-\tau)^{\gamma-1}}{\Gamma(\gamma)(1-\mathscr{B})} h(\tau) d \tau \\
= & -\int_{0}^{1} H(t, \tau) h(\tau) d \tau .
\end{aligned}
$$

Therefore,

$$
D^{\alpha} u(t)+\phi_{q}\left(\int_{0}^{1} H(t, \tau) h(\tau) d \tau\right)=0
$$

Combining with the boundary conditions $u(0)=0, D^{\beta} u(1)=a D^{\alpha} u(\xi)$, by Lemma 3.1, we obtain the unique solution of problem (3.9), (3.10)

$$
u(t)=\int_{0}^{1} G(t, s) \phi_{q}\left(\int_{0}^{1} H(s, \tau) h(\tau) d \tau\right) d s
$$

The proof is completed.

Lemma 3.3 Suppose $1-\mathscr{A}>0,1-\mathscr{B}>0$. The functions $G(t, s)$ and $H(t, s)$ have the following properties:

(1) $G(t, s), H(t, s) \in C([0,1] \times[0,1])$ and $G(t, s)>0, H(t, s)>0$ for $t, s \in(0,1)$;

(2) there exist functions $\omega, \omega_{1} \in C((0,1),(0,+\infty))$ such that

$$
\omega(s) \geq \max _{0 \leq t \leq 1} G(t, s), \quad \omega_{1}(s) \geq \max _{0 \leq t \leq 1} H(t, s)
$$


where

$$
\begin{aligned}
& g(t, s)=\frac{t^{\alpha-1}(1-s)^{\alpha-\beta-1}-(t-s)^{\alpha-1}}{\Gamma(\alpha)}, \\
& g_{1}(t, s)=\frac{[t(1-s)]^{\gamma-1}-(t-s)^{\gamma-1}}{\Gamma(\gamma)}, \\
& \omega(s)=g(s, s)+\frac{\mathscr{A}(1-s)^{\alpha-\beta-1}}{\Gamma(\alpha)(1-\mathscr{A})}, \quad \omega_{1}(s)=g_{1}(s, s)+\frac{\mathscr{B}(1-s)^{\gamma-1}}{\Gamma(\gamma)(1-\mathscr{B})}, \quad s \in(0,1) .
\end{aligned}
$$

Proof It is obvious that $g(t, s)>0, g_{1}(t, s)>0$ for $s, t \in(0,1)$. We note that $g(t, s), g_{1}(t, s)$ are decreasing with respect to $t$ for $s \leq t$ and increasing with respect to $t$ for $t \leq s$. Hence,

$$
\begin{array}{ll}
\max _{0 \leq t \leq 1} g(t, s)=g(s, s)=\frac{s^{\alpha-1}(1-s)^{\alpha-\beta-1}}{\Gamma(\alpha)}, & s \in(0,1) ; \\
\max _{0 \leq t \leq 1} g_{1}(t, s)=g_{1}(s, s)=\frac{s^{\gamma-1}(1-s)^{\gamma-1}}{\Gamma(\gamma)}, & s \in(0,1) .
\end{array}
$$

We first prove the statement (1). From the definitions of $G(t, s)$ and $H(t, s)$, it is clear that $G(t, s), H(t, s) \in C([0,1] \times[0,1])$.

For $0<s \leq t<1, s \leq \xi$, we have

$$
\begin{aligned}
G(t, s)= & \frac{t^{\alpha-1}(1-s)^{\alpha-\beta-1}-(1-\mathscr{A})(t-s)^{\alpha-1}-a t^{\alpha-1}(\xi-s)^{\alpha-\beta-1}}{\Gamma(\alpha)(1-\mathscr{A})} \\
= & \frac{1}{\Gamma(\alpha)}\left(1+\frac{\mathscr{A}}{1-\mathscr{A}}\right) t^{\alpha-1}(1-s)^{\alpha-\beta-1}-\frac{(t-s)^{\alpha-1}}{\Gamma(\alpha)} \\
& -\frac{a t^{\alpha-1}(\xi-s)^{\alpha-\beta-1}}{\Gamma(\alpha)(1-\mathscr{A})} \\
= & \frac{t^{\alpha-1}(1-s)^{\alpha-\beta-1}-(t-s)^{\alpha-1}}{\Gamma(\alpha)} \\
& +\frac{a t^{\alpha-1}\left[\xi^{\alpha-\beta-1}(1-s)^{\alpha-\beta-1}-(\xi-s)^{\alpha-1}\right]}{\Gamma(\alpha)(1-\mathscr{A})} \\
\geq & g(t, s)+\frac{a t^{\alpha-1}}{\Gamma(\alpha)(1-\mathscr{A})} g(\xi, s) \\
> & 0 .
\end{aligned}
$$

By using an analogous argument, we have $G(t, s)>0$ for $0<\xi \leq s \leq t<1$ or $0<t \leq s \leq \xi<1$ or $0<t \leq s<1, \xi \leq s$.

Hence, $G(t, s)>0$ for $t, s \in(0,1)$.

For $0<s \leq t<1, s \leq \eta$, we have

$$
\begin{aligned}
H(t, s) & =\frac{[t(1-s)]^{\gamma-1}-b^{p-1}[t(\eta-s)]^{\gamma-1}-(1-\mathscr{B})(t-s)^{\gamma-1}}{\Gamma(\gamma)(1-\mathscr{B})} \\
& =\left(1+\frac{\mathscr{B}}{1-\mathscr{B}}\right) \frac{[t(1-s)]^{\gamma-1}}{\Gamma(\gamma)}-\frac{(t-s)^{\gamma-1}}{\Gamma(\gamma)}-\frac{b^{p-1}[t(\eta-s)]^{\gamma-1}}{\Gamma(\gamma)(1-\mathscr{B})}
\end{aligned}
$$




$$
\begin{aligned}
& =\frac{[t(1-s)]^{\gamma-1}-(t-s)^{\gamma-1}}{\Gamma(\gamma)}+\frac{b^{p-1} t^{\gamma-1}\left[\eta^{\gamma-1}(1-s)^{\gamma-1}-(\eta-s)^{\gamma-1}\right]}{\Gamma(\gamma)(1-\mathscr{B})} \\
& =g_{1}(t, s)+\frac{b^{p-1} t^{\gamma-1}}{1-\mathscr{B}} g_{1}(\eta, s) \\
& >0 .
\end{aligned}
$$

Similarly, $H(t, s)>0$ for $0<\eta \leq s \leq t<1$ or $0<t \leq s \leq \eta<1$ or $0<t \leq s<1, \eta \leq s$.

Hence, $H(t, s)>0$ for $t, s \in(0,1)$.

Now follows the proof of the statement (2).

For $0 \leq s \leq t \leq 1, s \leq \xi$, one has

$$
\begin{aligned}
& \max _{0 \leq t \leq 1} G(t, s) \\
& \quad=\max _{0 \leq t \leq 1}\left(g(t, s)+\frac{a t^{\alpha-1}\left[\xi^{\alpha-\beta-1}(1-s)^{\alpha-\beta-1}-(\xi-s)^{\alpha-1}\right]}{\Gamma(\alpha)(1-\mathscr{A})}\right) \\
& \quad \leq g(s, s)+\frac{\mathscr{A}(1-s)^{\alpha-\beta-1}}{\Gamma(\alpha)(1-\mathscr{A})}=\omega(s) .
\end{aligned}
$$

For $0<\xi \leq s \leq t \leq 1$, one has

$$
\begin{aligned}
& \max _{0 \leq t \leq 1} G(t, s) \\
& =\max _{0 \leq t \leq 1} \frac{t^{\alpha-1}(1-s)^{\alpha-\beta-1}-(1-\mathscr{A})(t-s)^{\alpha-1}}{\Gamma(\alpha)(1-\mathscr{A})} \\
& \quad=\max _{0 \leq t \leq 1}\left(\frac{1}{\Gamma(\alpha)}\left(1+\frac{\mathscr{A}}{1-\mathscr{A}}\right) t^{\alpha-1}(1-s)^{\alpha-\beta-1}-\frac{(t-s)^{\alpha-1}}{\Gamma(\alpha)}\right) \\
& \quad \leq g(s, s)+\frac{\mathscr{A}(1-s)^{\alpha-\beta-1}}{\Gamma(\alpha)(1-\mathscr{A})}=\omega(s) .
\end{aligned}
$$

For $0 \leq t \leq s \leq \xi<1$,

$$
\begin{aligned}
& \max _{0 \leq t \leq 1} G(t, s) \\
& =\max _{0 \leq t \leq 1} \frac{t^{\alpha-1}(1-s)^{\alpha-\beta-1}-a t^{\alpha-1}(\xi-s)^{\alpha-\beta-1}}{\Gamma(\alpha)(1-\mathscr{A})} \\
& =\max _{0 \leq t \leq 1}\left(\frac{t^{\alpha-1}(1-s)^{\alpha-\beta-1}}{\Gamma(\alpha)}+\frac{a t^{\alpha-1}\left[(\xi-\xi s)^{\alpha-\beta-1}-(\xi-s)^{\alpha-1}\right]}{\Gamma(\alpha)(1-\mathscr{A})}\right) \\
& \quad \leq g(s, s)+\frac{\mathscr{A}(1-s)^{\alpha-\beta-1}}{\Gamma(\alpha)(1-\mathscr{A})}=\omega(s) .
\end{aligned}
$$

For $0 \leq t \leq s \leq 1, \xi \leq s$,

$$
\begin{aligned}
\max _{0 \leq t \leq 1} G(t, s) & =\max _{0 \leq t \leq 1} \frac{t^{\alpha-1}(1-s)^{\alpha-\beta-1}}{\Gamma(\alpha)(1-\mathscr{A})} \\
& =\max _{0 \leq t \leq 1}\left(\frac{1}{\Gamma(\alpha)}\left(1+\frac{\mathscr{A}}{1-\mathscr{A}}\right) t^{\alpha-1}(1-s)^{\alpha-\beta-1}\right) \\
& \leq g(s, s)+\frac{\mathscr{A}(1-s)^{\alpha-\beta-1}}{\Gamma(\alpha)(1-\mathscr{A})}=\omega(s) .
\end{aligned}
$$


So,

$$
\max _{0 \leq t \leq 1} G(t, s) \leq \omega(s), \quad s \in(0,1) .
$$

For $0 \leq s \leq t \leq 1, s \leq \eta$, one has

$$
\begin{aligned}
\max _{0 \leq t \leq 1} H(t, s) & \leq \max _{0 \leq t \leq 1}\left(g_{1}(t, s)+\frac{b^{p-1} t^{\gamma-1}}{1-\mathscr{B}} g_{1}(\eta, s)\right) \\
& \leq g_{1}(s, s)+\frac{\mathscr{B}(1-s)^{\gamma-1}}{\Gamma(\gamma)(1-\mathscr{B})}=\omega_{1}(s) .
\end{aligned}
$$

For $0<\eta \leq s \leq t \leq 1$, one has

$$
\begin{aligned}
\max _{0 \leq t \leq 1} H(t, s) \\
=\max _{0 \leq t \leq 1} \frac{[t(1-s)]^{\gamma-1}-(1-\mathscr{B})(t-s)^{\gamma-1}}{\Gamma(\gamma)(1-\mathscr{B})} \\
=\max _{0 \leq t \leq 1}\left(g_{1}(t, s)+\frac{\mathscr{B} t^{\gamma-1}(1-s)^{\gamma-1}}{\Gamma(\gamma)(1-\mathscr{B})}\right) \\
\quad \leq g_{1}(s, s)+\frac{\mathscr{B}(1-s)^{\gamma-1}}{\Gamma(\gamma)(1-\mathscr{B})}=\omega_{1}(s) .
\end{aligned}
$$

For $0 \leq t \leq s \leq \eta<1$,

$$
\begin{aligned}
\max _{0 \leq t \leq 1} H(t, s) & =\max _{0 \leq t \leq 1} \frac{[t(1-s)]^{\gamma-1}-b^{p-1}[t(\eta-s)]^{\gamma-1}}{\Gamma(\gamma)(1-\mathscr{B})} \\
& =\max _{0 \leq t \leq 1}\left(\frac{[t(1-s)]^{\gamma-1}}{\Gamma(\gamma)}+\frac{b^{\gamma-1} t^{\gamma-1}}{1-\mathscr{B}} g_{1}(\eta, s)\right) \\
& \leq g_{1}(s, s)+\frac{\mathscr{B}(1-s)^{\gamma-1}}{\Gamma(\gamma)(1-\mathscr{B})}=\omega_{1}(s) .
\end{aligned}
$$

For $0 \leq t \leq s \leq 1, \eta \leq s$,

$$
\begin{aligned}
\max _{0 \leq t \leq 1} H(t, s) & =\max _{0 \leq t \leq 1} \frac{[t(1-s)]^{\gamma-1}}{\Gamma(\gamma)(1-\mathscr{B})} \\
& =\max _{0 \leq t \leq 1}\left(\frac{[t(1-s)]^{\gamma-1}}{\Gamma(\gamma)}+\frac{\mathscr{B}[t(1-s)]^{\gamma-1}}{\Gamma(\gamma)(1-\mathscr{B})}\right) \\
& \leq g_{1}(s, s)+\frac{\mathscr{B}(1-s)^{\gamma-1}}{\Gamma(\gamma)(1-\mathscr{B})}=\omega_{1}(s) .
\end{aligned}
$$

So,

$$
\max _{0 \leq t \leq 1} H(t, s) \leq \omega_{1}(s), \quad s \in(0,1)
$$

It is obvious that $\omega(s), \omega_{1}(s) \in C((0,1),(0,+\infty))$. The proof is completed.

Let $E=C[0,1]$ be a Banach space with the maximum norm $\|u\|=\max _{0 \leq t \leq 1}|u(t)|$. Define the cone $P \subset E$ by $P=\{u \in E \mid u(t) \geq 0,0 \leq t \leq 1\}$. 
Lemma 3.4 Let $T: P \rightarrow$ E be the operator defined by

$$
(T u)(t)=\int_{0}^{1} G(t, s) \phi_{q}\left(\int_{0}^{s} H(s, \tau) f(\tau, u(\tau)) d \tau\right) d s .
$$

Then $T: P \rightarrow P$ is completely continuous.

Proof The operator $T: P \rightarrow P$ is continuous in view of nonnegative and continuity of $G(t, s), H(t, s)$ and $f(t, u)$. Furthermore it is easy see by the Arzela-Ascoli theorem and Lebesgue dominated convergence theorem that $T: P \rightarrow P$ is completely continuous.

For convenience, we introduce the following notation:

$$
L=\left[\int_{0}^{1} \omega(s) \phi_{q}\left(\int_{0}^{s} \omega_{1}(\tau) d \tau\right) d s\right]^{-1}
$$

Theorem 3.1 Assume there exists a constant $\lambda>0$ such that

$\left(C_{1}\right) f\left(t, x_{1}\right) \leq f\left(t, x_{2}\right)$ for any $0 \leq t \leq 1,0 \leq x_{1} \leq x_{2} \leq \lambda$;

$\left(C_{2}\right) \max _{0 \leq t \leq 1} f(t, \lambda) \leq \phi_{p}(\lambda L)$;

$\left(C_{3}\right) f(t, 0) \neq 0$ for $0 \leq t \leq 1$.

Then problem (1.1), (1.2) has two positive solution $u^{*}$ and $v^{*}$, such that

$0<\left\|u^{*}\right\| \leq \lambda$ and $\lim _{n \rightarrow \infty} T^{n} u_{0}=u^{*}$, where $u_{0}(t)=\lambda$,

$0<\left\|v^{*}\right\| \leq \lambda$ and $\lim _{n \rightarrow \infty} T^{n} v_{0}=v^{*}$, where $v_{0}(t)=0$.

Proof Define $P_{\lambda}=\{u \in P \mid\|u\| \leq \lambda\}$. In what follows, we first prove $T P_{\lambda} \subseteq P_{\lambda}$.

Let $u \in P_{\lambda}$, then $0 \leq u(t) \leq\|u\| \leq \lambda$. By assumption $\left(C_{1}\right)$ and $\left(C_{2}\right)$, we have

$$
0 \leq f(t, u(t)) \leq f(t, \lambda) \leq \phi_{p}(\lambda L)
$$

For any $u \in P_{\lambda}$, by Lemma 3.4, we know that $T u \in P$, and as a result

$$
\begin{aligned}
\|T u\| & =\max _{0 \leq t \leq 1} \int_{0}^{1} G(t, s) \phi_{q}\left(\int_{0}^{s} H(s, \tau) f(\tau, u(\tau)) d \tau\right) d s \\
& \leq \int_{0}^{1} \omega(s) \phi_{q}\left(\int_{0}^{s} \phi_{p}(\lambda L) \omega_{1}(\tau) d \tau\right) d s \\
& =\lambda L \int_{0}^{1} \omega(s) \phi_{q}\left(\int_{0}^{s} \omega_{1}(\tau) d \tau\right) d s \\
& =\lambda .
\end{aligned}
$$

Hence $T u \in P_{\lambda}$. Thus, we get $T P_{\lambda} \subseteq P_{\lambda}$.

Let $u_{0}(t)=\lambda, 0 \leq t \leq 1$, then $\left\|u_{0}\right\|=\lambda$ and $u_{0} \in P_{\lambda}$. Let $u_{1}(t)=T u_{0}(t)$, then $u_{1} \in P_{\lambda}$. Define

$$
u_{n+1}=T u_{n}=T^{n+1} u_{0}, \quad n=0,1,2, \ldots
$$

Since $T P_{\lambda} \subseteq P_{\lambda}$, one has $u_{n} \in P_{\lambda}(n=0,1,2, \ldots)$. From Lemma 3.3, $T$ is compact; we assert that $\left\{u_{n}\right\}_{n=1}^{\infty}$ has a convergent subsequence $\left\{u_{n_{k}}\right\}_{k=1}^{\infty}$ and there exists $u^{*} \in P_{\lambda}$ such that 
$u_{n_{k}} \rightarrow u^{*}$. From the definition of $T$ and $\left(C_{1}\right)$, for any $t \in[0,1]$, we have

$$
\begin{aligned}
u_{1}(t) & =\left(T u_{0}\right)(t) \\
& =\int_{0}^{1} G(t, s) \phi_{q}\left(\int_{0}^{s} H(s, \tau) f\left(\tau, u_{0}(\tau)\right) d \tau\right) d s \\
& \leq \int_{0}^{1} \omega(s) \phi_{q}\left(\int_{0}^{s} \phi_{p}(\lambda L) \omega_{1}(\tau) d \tau\right) d s \\
& =\lambda L \int_{0}^{1} \omega(s) \phi_{q}\left(\int_{0}^{s} \omega_{1}(\tau) d \tau\right) d s \\
& =\lambda \\
& =u_{0}(t) .
\end{aligned}
$$

So,

$$
u_{2}(t)=T u_{1}(t) \leq T u_{0}(t)=u_{1}(t), \quad 0 \leq t \leq 1 .
$$

Hence, by the induction we have

$$
u_{n+1} \leq u_{n}, \quad 0 \leq t \leq 1, n=0,1,2, \ldots
$$

Thus, there exists $u^{*} \in P_{\lambda}$ such that $u_{n} \rightarrow u^{*}$. Applying the continuity of $T$ and $u_{n+1}=T u_{n}$, we get $T u^{*}=u^{*}$.

Let $v_{0}=0,0 \leq t \leq 1$, then $v_{0} \in P_{\lambda}$. Let $v_{1}=T v_{0}$, then $v_{1} \in P_{\lambda}$. Define

$$
v_{n+1}=T v_{n}=T^{n+1} v_{0}, \quad n=0,1,2, \ldots
$$

Since $T: P_{\lambda} \rightarrow P_{\lambda}$, we have $v_{n} \subseteq P_{\lambda}, n=0,1,2, \ldots$. Since $T$ is completely continuous, we assert that $\left\{v_{n}\right\}_{n=1}^{\infty}$ is a sequentially compact set.

Since $v_{1}(t)=T v_{0}(t)=(T 0)(t) \geq 0,0 \leq t \leq 1$, one has

$$
v_{2}(t)=T v_{1}(t) \geq(T 0)(t)=v_{1}(t), \quad 0 \leq t \leq 1 .
$$

Hence, by the induction we have

$$
v_{n+1} \geq v_{n}, \quad 0 \leq t \leq 1, n=0,1,2, \ldots
$$

Thus, there exists $v^{*} \in P_{\lambda}$ such that $v_{n} \rightarrow v^{*}$. Applying the continuity of $T$ and $v_{n+1}=T v_{n}$, we get $T v^{*}=v^{*}$.

It is well known that each fixed point of operator $T$ in $P$ is a solution of problem (1.1). Furthermore, if $f(t, 0) \neq 0,0 \leq t \leq 1$, then the zero function is not the solution of problem (1.1). Hence, we have $\left\|u^{*}\right\|>0,\left\|v^{*}\right\|>0$. Then $u^{*}$ and $v^{*}$ are two positive solutions of problem (1.1). The proof is completed.

By applying Theorem 3.1, we can get the following corollary. 
Corollary 3.1 Assume $\left(C_{3}\right)$ holds, and the following conditions hold:

( $\left.C_{4}\right) f\left(t, x_{1}\right) \leq f\left(t, x_{2}\right)$ for any $0 \leq t \leq 1,0 \leq x_{1} \leq x_{2}$;

$\left(C_{5}\right) \lim _{x \rightarrow \infty} \max _{0 \leq t \leq 1} \frac{f(t, x)}{x^{p-1}} \leq \phi_{p}(L)$ (particularly $\left.\lim _{x \rightarrow \infty} \max _{0 \leq t \leq 1} \frac{f(t, x)}{x^{p-1}}=0\right)$.

Then problem (1.1) have two positive solutions $u^{*}$ and $v^{*}$.

\section{Example}

Let $p=\frac{3}{2}, \alpha=\frac{4}{3}, \gamma=\frac{3}{2}, \beta=\frac{1}{4}, \xi=\frac{1}{3}, \eta=\frac{1}{4}, a=b=\frac{1}{2}$. We consider the following boundary value problem:

$$
\left\{\begin{array}{l}
D^{\frac{3}{2}}\left(\phi_{\frac{3}{2}}\left(D^{\frac{4}{3}} x(t)\right)\right)=f(t, x(t)), \quad 0<t<1, \\
x(0)=D^{\frac{4}{3}} x(0)=0, \quad D^{\frac{1}{4}} x(1)=\frac{1}{2} D^{\frac{1}{4}} x\left(\frac{1}{3}\right), \quad D^{\frac{4}{3}} x(1)=\frac{1}{2} D^{\frac{4}{3}} x\left(\frac{1}{4}\right),
\end{array}\right.
$$

where

$$
f(t, x)=\frac{1}{24}\left(1+x e^{t}+x^{\frac{3}{2}}\right)
$$

It is obvious that

$$
1-a \xi^{\alpha-\beta-1}=1-\frac{1}{2}\left(\frac{1}{3}\right)^{\frac{1}{12}}>0, \quad 1-b^{p-1} \eta^{\gamma-1}=1-\frac{1}{2^{\frac{3}{2}}}>0 .
$$

By computation, we can obtain $L \approx 0.2318$. We choose $\lambda=5$. So, $f(t, x)$ satisfies

(1) $f\left(t, x_{1}\right) \leq f\left(t, x_{2}\right)$ for any $0 \leq t \leq 1,0 \leq x_{1} \leq x_{2} \leq 5$;

(2) $\max _{0 \leq t \leq 1} f(t, \lambda)=f(1,5) \approx \frac{25.7703}{24} \approx 1.0738<\phi_{\frac{3}{2}}(\lambda L) \approx 1.0766$;

(3) $f(t, 0)=\frac{1}{24} \neq 0$ for $0 \leq t \leq 1$.

Then applying Theorem 3.1, problem (4.1) has two positive solutions $u^{*}$ and $v^{*}$ such that

$$
\begin{aligned}
& 0<\left\|u^{*}\right\| \leq 5 \text { and } \lim _{n \rightarrow \infty} T^{n} u_{0}=u^{*}, \text { where } u_{0}(t)=5, \\
& 0<\left\|v^{*}\right\| \leq 5 \text { and } \lim _{n \rightarrow \infty} T^{n} v_{0}=v^{*}, \text { where } v_{0}(t)=0
\end{aligned}
$$

\section{Acknowledgements}

Not applicable.

\section{Funding}

This work is supported by the Scientific Research Foundation of Hunan Provincial Education Department (16A198,

18C1019), National Natural Science Foundation of China (11571207), and the Taishan Scholar project.

\section{Availability of data and materials}

Data sharing not applicable to this article as no data sets were generated or analysed during the current study.

\section{Competing interests}

The authors declare that they have no competing interests.

\section{Authors' contributions}

All authors contributed equally to the writing of this paper. All authors read and approved the final manuscript.

\section{Authors' information}

Yuanshen Tian, professor, his main research field is fractional differential equations and boundary value problems. Zhanbing Bai, professor, his main research field is fractional differential equations and boundary value problems. Sujing Sun, doctoral candidate, the research fields is the application of nonlinear functional analysis on differential equations.

\section{Author details}

${ }^{1}$ College of Mathematics and Finance, Xiangnan University, Chenzhou, P.R. China. ${ }^{2}$ College of Mathematics and System Sciences, Shandong University of Science and Technology, Qingdao, P.R. China. 


\section{Publisher's Note}

Springer Nature remains neutral with regard to jurisdictional claims in published maps and institutional affiliations.

\section{Received: 11 April 2019 Accepted: 31 July 2019 Published online: 20 August 2019}

\section{References}

1. Agarwal, R.P., Baleanu, D., Hedayati, V., Rezapour, S.: Two fractional derivative inclusion problems via integral boundary condition. Appl. Math. Comput. 257, 205-212 (2015)

2. Atangana, A., Baleanu, D.: Caputo-Fabrizio derivative applied to groundwater flow within confined aquifer. J. Eng. Mech. 143(5), D4016005 (2017)

3. Aydogan, S.M., Baleanu, D., Mousalou, A., Rezapour, S.: On approximate solutions for two higher-order Caputo-Fabrizio fractional integro-differential equations. Adv. Differ. Equ. 2017, 221 (2017)

4. Aydogan, S.M., Baleanu, D., Mousalou, A., Shahram, R.: On high order fractional integro-differential equations including the Caputo-Fabrizio derivative. Bound. Value Probl. 2018, 90 (2018)

5. Bai, Z.: Solvability for a class of fractional $m$-point boundary value problem at resonance. Comput. Math. Appl. 62 , 1292-1302 (2011)

6. Bai, Z., Chen, Y., Lian, H., Sun, S.: On the existence of blow up solutions for a class of fractional differential equations. Fract. Calc. Appl. Anal. 17(4), 1175-1187 (2014)

7. Bai, Z., Zhang, S., Sun, S., Yin, C.: Monotone iterative method for fractional differential equations. Electron. J. Differ. Equ. 2016, 6 (2016)

8. Bai, Z., Zhang, Y.: Solvability of fractional three-point boundary value problems with nonlinear growth. Appl. Math. Comput. 218, 1719-1725 (2011)

9. Baleanu, D., Mousalou, A., Rezapour, S.: A new method for investigating approximate solutions of some fractional integro-differential equations involving the Caputo-Fabrizio derivative. Adv. Differ. Equ. 2017, 51 (2017)

10. Baleanu, D., Mousalou, A., Rezapour, S.: On the existence of solutions for some infinite coefficient-symmetric Caputo-Fabrizio fractional integro-differential equations. Bound. Value Probl. 2017, 145 (2017)

11. Baleanu, D., Mousalou, A., Rezapour, S.: The extended fractional Caputo-Fabrizio derivative of order $0 \leq \sigma<1$ on $C_{R}[0,1]$ and the existence of solutions for two higher-order series-type differential equations. Adv. Differ. Equ. 2018 255 (2018)

12. Baleanu, D., Rezapour, S., Mohammadi, H.: Some existence results on nonlinear fractional differential equations. Philos. Trans. R. Soc. 371, 20120144 (2013)

13. Baleanu, D., Rezapour, S., Saberpour, Z.: On fractional integro-differential inclusions via the extended fractional Caputo-Fabrizio derivation. Bound. Value Probl. 2019, 79 (2019)

14. Cabada, A., Wang, G.: Positive solutions of nonlinear fractional differential equations with integral boundary value conditions. Abstr. Appl. Anal. 2012, 403 (2012)

15. Chen, C., Song, H., Yang, H.: Liouville type theorems for stable solutions of $p$-Laplace equation in $R^{n}$. Nonlinear Anal. $160,44-52(2017)$

16. Chen, F., Baleanu, D., Wu, G.: Existence results of fractional differential equations with Riesz-Caputo derivative. Eur. Phys. J. Spec. Top. 226, 3411-3425 (2017)

17. Cui, Y.: Uniqueness of solution for boundary value problems for fractional differential equations. Appl. Math. Lett. 51, 48-54 (2016)

18. Cui, Y., Sun, J.: A generalization of Mahadevan's version of the Krein-Rutman theorem and applications to $p$-Laplacian boundary value problems. Abstr. Appl. Anal. 2012, 1 (2012)

19. Dong, H., Guo, B., Yin, B.: Generalized fractional supertrace identity for Hamiltonian structure of NLS-MKdV hierarchy with self-consistent sources. Anal. Math. Phys. 6, 199-209 (2016)

20. Dong, X., Bai, Z., Zhang, S.: Positive solutions to boundary value problems of $p$-Laplacian with fractional derivative. Bound. Value Probl. 2017, 1 (2017)

21. Feng, H., Pang, H., Ge, W.: Multiplicity of symmetric Positive solutions fora multipoint boundary problem with a one-dimensional p-Laplacian. Nonlinear Anal. 69, 3050-3059 (2008)

22. Feng, M., Li, P., Sun, S.: Symmetric positive solutions for fourth-order $n$-dimensional $m$-Laplace systems. Bound. Value Probl. (2018). https://doi.org/10.1186/s13661-018-0981-3

23. Firoozjaee, M., Jafari, H., Lia, A., Baleanu, D.: Numerical approach of Fokker-Planck equation with Caputo-Fabrizio fractional derivative using Ritz approximation. J. Comput. Appl. Math. 339, 367-373 (2018)

24. Fu, C., Lu, C., Yang, H.: Time-space fractional $(2+1)$ dimensional nonlinear Schrodinger equation for envelope gravity waves in baroclinic atmosphere and conservation laws as well as exact solutions. Adv. Differ. Equ. 2018, 56 (2018)

25. Gu, C., Wu, G.: Positive solutions of fractional differential equations with the Riesz space derivative. Appl. Math. Lett. 95, 59-64 (2019)

26. Guo, M., Fu, C., Zhang, Y., Liu, J., Yang, H.: Study of ion-acoustic solitary waves in a magnetized plasma using the three-dimensional time-space fractional Schamel-KdV equation. Complexity (2018). https://doi.org/10.1155/2018/6852548

27. Guo, X., Zhang, G., Li, H.: Fixed point theorems for Meir-Keeler condensing nonself-mappings with an application. J. Fixed Point Theory Appl. 20, 33 (2018)

28. Hao, X., Wang, H., Liu, L., Cui, Y.: Positive solutions for a system of nonlinear fractional nonlocal boundary value problems with parameters and p-Laplacian operator. Bound. Value Probl. 2017, 182 (2017)

29. He, L., Dong, X., Bai, Z., Chen, B.: Solvability of some two-point fractional boundary value problems under barrier strip conditions. J. Funct. Spaces 2017, Article ID 1465623 (2017)

30. Ji, D., Bai, Z., Ge, W.: The existence of countably many positive solutions for singular multipoint boundary value problems. Nonlinear Anal. 72, 955-964 (2010)

31. Jiang, C., Zhang, F., Li, T.: Synchronization and anti-synchronization of N-coupled fractional-order complex systems with ring connection. Math. Methods Appl. Sci. 41, 2625-2638 (2018)

32. Kilbas, A., Srivastava, H., Trujillo, J.: Theory and Applications of Fractional Differential Equations. Elsevier, Amsterdam (2006) 
33. Kojabad, E.A., Rezapour, S.: Approximate solutions of a sum-type fractional integro-differential equation by using Chebyshev and Legendre polynomials. Adv. Differ. Equ. 2017, 351 (2017)

34. Lakshmikantham, V., Vatsala, A.S.: General uniqueness and monotone iterative technique for fractional differential equations. Appl. Math. Lett. 21, 828-834 (2008)

35. Li, H., Sun, J.: Positive solutions of superlinear semipositone nonlinear boundary value problems. Comput. Math. Appl. 61, 2806-2815 (2011)

36. Lian, H., Wang, D., Bai, Z., Agarwal, R.: Periodic and subharmonic solutions for a class of second-order $p$-Laplacian Hamiltonian systems. Bound. Value Probl. (2014). https://doi.org/10.1186/s13661-014-0260-x

37. Liu, F.: A note of Littlewood-Paley functions on Triebel-Lizorkin spaces. Bull. Korean Math. Soc. 55(2), 659-672 (2018)

38. Liu, F., Wu, H., Zhang, D.: Lp bounds for parametric Marcinkiewicz integrals with mixed homogeneity. Math. Inequal. Appl. 18(2), 453-469 (2015)

39. Liu, X., Jia, M.: The method of lower and upper solutions for the general boundary value problems of fractional differential equations with $p$-Laplacian. Adv. Differ. Equ. 2018, 28 (2018)

40. Liu, X., Jia, M.: Solvability and numerical simulations for BVPs of fractional coupled systems involving left and right fractional derivatives. Appl. Math. Comput. 353, 230-242 (2019)

41. Liu, X., Li, Y., Zhang, W.: Stochastic linear quadratic optimal control with constraint for discrete-time systems. Appl. Math. Comput. 228, 264-270 (2014)

42. Lu, C., Fu, C., Yang, H.: Time-fractional generalized Boussinesq equation for Rossby solitary waves with dissipation effect in stratified fluid and conservation laws as well as exact solutions. Appl. Math. Comput. 327, 104-116 (2018)

43. LV, W., Wang, F.: Adaptive tracking control for a class of uncertain nonlinear systems with infinite number of actuator failures using neural networks. Adv. Differ. Equ. 2017, 374 (2017)

44. Salem, H.A.H.: On the fractional order $m$-point boundary value problem in reflexive Banach spaces and weak topologies. J. Comput. Appl. Math. 224, 567-572 (2009)

45. Sheng, K., Zhang, W., Bai, Z:: Positive solutions to fractional boundary value problems with $p$-Laplacian on time scales, Bound. Value Probl. (2018). https://doi.org/10.1186/s13661-018-0990-2

46. Song, Q., Bai, Z:: Positive solutions of fractional differential equations involving the Riemann-Stieltjes integral boundary condition. Adv. Differ. Equ. (2018). https://doi.org/10.1186/s13662-018-1633-8

47. Su, H., Wei, Z., Wang, B.: The existence of positive solutions for a nonlinear four-point singular boundary value problem with a $p$-Laplacian operator. Nonlinear Anal. 66, 2204-2217 (2007)

48. Tian, Y., Bai, Z:: Existence results for the three-point impulsive boundary value problem involving fractional differential equations. Comput. Math. Appl. 59, 2601-2609 (2010)

49. Tian, Y., Sun, S., Bai, Z.: Positive solutions of fractional differential equations with $p$-Laplacian. J. Funct. Spaces 2017 Article ID 3187492 (2017)

50. Tian, Y., Wei, Y., Sun, S.: Multiplicity for fractional differential equations with $p$-Laplacian. Bound. Value Probl. (2018). https://doi.org/10.1186/s13661-018-1049-0

51. Wang, G., Agarwal, R., Cabada, A.: Existence results and monotone iterative technique for systems of nonlinear fractional differential equations. Appl. Math. Lett. 25(6), 1019-1024 (2012)

52. Wang, Y., Hou, C.: Existence of multiple positive solutions for one dimensional $p$-Laplacian. J. Math. Anal. Appl. 315, 144-153 (2006)

53. Wang, Z.: A numerical method for delayed fractional-order differential equations. J. Appl. Math. 2013, Article ID 256071 (2013)

54. Wang, Z., Huang, X., Shi, G.: Analysis of nonlinear dynamics and chaos in a fractional order financial system with time delay. Comput. Math. Appl. 62, 1531-1539 (2011)

55. Wang, Z., Wang, X., Li, Y., Huang, X.: Stability and Hopf bifurcation of fractional-order complex valued single neuron model with time delay. Int. J. Bifurc. Chaos 27, 1750209 (2017)

56. Wang, Z., Xie, Y., Lu, J., Li, Y.: Stability and bifurcation of a delayed generalized fractional-order prey-predator model with interspecific competition. Appl. Math. Comput. 347, 360-369 (2019)

57. Wei, Y., Bai, Z.: Solvability of some fractional boundary value problems with a convection term. Discrete Dyn. Nat. Soc 2019, Article ID 1230502 (2019). https://doi.org/10.1155/2019/1230502

58. Wei, Y., Bai, Z., Sun, S.: On positive solutions for some second-order three-point boundary value problems with convection term. J. Inequal. Appl. 2019, 72 (2019). https://doi.org/10.1186/s13660-019-2029-3

59. Wei, Y., Song, Q., Bai, Z.: Existence and iterative method for some fourth order nonlinear boundary value problems. Appl. Math. Lett. 87, 101-107 (2019)

60. Wu, J., Zhang, X., Liu, L., Wu, Y., Cui, Y.: The convergence analysis and error estimation for unique solution of a p-Laplacian fractional differential equation with singular decreasing nonlinearity. Bound. Value Probl. 2018, 82 (2018)

61. Zhai, C., Wang, W., Li, H.: A uniqueness method to a new Hadamard fractional differential system with four-point boundary conditions. J. Inequal. Appl. 2018, 207 (2018)

62. Zhang, J., Zhang, G., Li, H.: Positive solutions of second-order problem with dependence on derivative in nonlinearity under Stieltjes integral boundary condition. Electron. J. Qual. Theory Differ. Equ. (2018) https://doi.org/10.14232/ejqtde.2018.1.4

63. Zhang, S.: Positive solutions to singular boundary value problem for nonlinear fractional differential equation. Comput. Math. Appl. 59, 1300-1309 (2009)

64. Zhang, W., Bai, Z., Sun, S.: Extremal solutions for some periodic fractional differential equations. Adv. Differ. Equ. 2016, 1 (2016)

65. Zhang, X., Cui, Y.: Positive solutions for fourth-order singular $p$-Laplacian differential equations with integral boundary conditions. Bound. Value Probl. (2010). https://doi.org/10.1155/2010/862079

66. Zhang, X., Liu, L., Wu, Y., Cui, Y.: Entire blow-up solutions for a quasilinear $p$-Laplacian Schrödinger equation with a non-square diffusion term. Appl. Math. Lett. 74, 85-93 (2017)

67. Zhang, Y.: Existence results for a coupled system of nonlinear fractional multi-point boundary value problems at resonance. J. Inequal. Appl. (2018). https://doi.org/10.1186/s13660-018-1792-X

68. Zhong, Q., Zhang, X., Lu, X., Fu, Z.: Uniqueness of successive positive solution for nonlocal singular higher-order fractional differential equations involving arbitrary derivatives. J. Funct. Spaces 2018, Article ID 6207682 (2018) 
69. Zou, Y., Cui, Y.: Existence results for a functional boundary value problem of fractional differential equations. Adv. Differ. Equ. 2013, 233 (2013)

70. Zou, Y., He, G.: Fixed point theorem for systems of nonlinear operator equations and applications to $\left(p_{1}, p_{2}\right)$-Laplacian system. Mediterr. J. Math. (2018). https://doi.org/10.1007/s00009-018-1119-7

71. Zou, Y., Liu, L., Cui, Y.: The existence of solutions for four-point coupled boundary value problems of fractional differential equations at resonance. Abstr. Appl. Anal. 2014, Article ID 314083 (2014)

72. Zuo, M., Hao, X., Liu, L., Cui, Y.: Existence results for impulsive fractional integro-differential equation of mixed type with constant coefficient and antiperiodic boundary conditions. Bound. Value Probl. 2017, 161 (2017)

Submit your manuscript to a SpringerOpen ${ }^{\circ}$ journal and benefit from:

- Convenient online submission

- Rigorous peer review

- Open access: articles freely available online

- High visibility within the field

- Retaining the copyright to your article

Submit your next manuscript at $\boldsymbol{~ s p r i n g e r o p e n . c o m ~}$ 